\title{
APTIDÃO FÍSICA RELACIONADA À SAÚDE E HÁBITOS DE VIDA EM ESCOLARES DE 10 A 14 ANOS
}

\section{Physical Fitness Related to Health and Life Style in Scholar's of 10 to 14 Years Old}

\author{
Rafael Abeche Generosi ${ }^{1}{ }^{12}$; Daniel Garlipp ${ }^{1}$; Fernando Braga ${ }^{1}$; Marcelo Cardoso ${ }^{1}$ \\ ${ }^{1}$ Escola Superior de Educação Física (ESEF), Programa de Pós-Graduação em Ciências do Movimento \\ Humano (PPGCMH), Universidade Federal do Rio Grande do Sul (UFRGS). \\ ${ }^{2}$ Bolsista CNPQ.
}

\begin{abstract}
Resumo: O objetivo do estudo foi descrever e explorar o perfil da aptidão física relacionada à saúde (ApFRS) e dos hábitos de vida (HbtV) em crianças e jovens escolares do municipio de Barra do Ribeiro/RS divididos por idades (10 a :14 anos) e sexo (rapazes e moças). A amostra intencional foi composta de 124 rapazes e 116 moças, totalizando 240 individuos. Inicialmente foi aplicado um questionário estruturado em três unidades (dados pessoais; tabagismo e etilismo; atividade fisica habitual); e posteriormente, as avaliaçōes fisicas: estatura, massa corporal, circunferência de cintura, dobras cutâneas do triceps e da perna, força/resistência abdominal, flexibilidade e resistência geral. Utilizaram-se tratamentos estatísticos de exploração (normalidade dos dados e verificação de outliers) e descrição (médias, desvios-padrões, freqüência de ocorrência). Observou-se um incremento nos valores médios da maioria das variáveis físicas, dos 10 para os 14 anos nos rapazes e nas moças, com exceção da flexibilidade. Nas dobras cutâneas do tríceps e da perna, e na atividade física habitual, não houve um padrão de incremento ou decréscimo. No tabagismo encontrou-se uma ocorrência de $8 \%$ (rapazes) e $6 \%$ (moças), e no etilismo 33\% (rapazes) e $27 \%$ (moças). Com o estudo foi possível conhecer em que estado se encontra uma razoável parcela de escolares do município em relação à ApFRS e HbtV; e os achados podem servir de base teórica para outros pesquisadores da área em discussão.
\end{abstract}

Palavras-chave: Aptidão Física, Atividade Fisica, Saúde.

\begin{abstract}
The aim objective of the study was to describe and to explore the profile of physical fitness related to health (PFRH) and life style (LS) in scholar's children and youth who lived in Barra do Ribeiro/RS, divided by ages (10 to 14 years old) and gender (boys and girls). The intentional sample was reached by 124 boys and 116 girls, totalizing 240 individuals. Initially, a questionnaire structuralized in three units was applied (personal data; tabagism and alcoholism; habitual physical activity); and later, the physical evaluations: stature, body mass, waist circumference, triceps and leg skinfolds, abdominal power/resistance, flexibility, general resistance. Statistical treatments of exploration (normality of the data and verification of outliers) and description (average, standard deviation, frequency of occurrence) had been used. Was observed an increment in average values of most the physical variables, the 10 to 14 years old and in both genders; with exception of flexibility variable. In triceps and leg skinfolds, and habitual physical activity, did not have a standard of increment or decrease. In tabagism was observed and occurrence of $8 \%$ (boys) and $6 \%$ (girls), and in alcoholism $33 \%$ (boys) and $27 \%$ (girls). With the study it was possible to know a parcel of the town scholar's in relation to PFRH and LS; and the findings can serve of theoretical base for other researchers of the prominence area.
\end{abstract}

Keywords: Physical Fitness, Physical Activity, Health.

Aceito em 18/02/2011 - Revista de Educação Física 2011 Abr; 151:3-10. Rio de Janeiro - Brasil

\section{INTRODUÇÃO}

Os estudos de caráter descritivo exploratório se distinguem por tratar situações que já estão apresentadas aos pesquisadores. Em outras palavras, não há uma efetiva intervenção experimental dos investigadores. Ele não planeja qualquer experimento para testar a sua hipótese. Não existe manipulação das variáveis. Portanto, este tipo de estudo se delimita a analisar determinados fenômenos, definir seus pressupostos e identificar suas estruturas. A finalidade principal do método descritivo explora- tório é proporcionar um perfil capaz de caracterizar de forma precisa as variáveis envolvidas em um determinado fenômeno, aceitando as limitações atribuídas pelas variáveis intervenientes ${ }^{(1-4)}$.

No caso do presente estudo, podem-se definir como fenômenos de investigação a aptidão física relacionada à saúde e os hábitos de vida. Em relação aos hábitos de vida, mais precisamente o nível de atividade física habitual, o número de horas de assistência de televisão, o hábito de fumar e o hábito de ingerir bebidas alcoólicas.

$\mathrm{Na}$ área das Ciências do Movimento Humano 
e da Saúde é de alta relevância estudar estes fenômenos, principalmente pelas suas relações com as doenças cardiovasculares (DCVs). Estudar estas variáveis em crianças e jovens escolares, então, é de fundamental importância, pelo fato de que já nestas faixas etárias os estudos têm evidenciado um elevado número de portadores de fatores de risco para as $\mathrm{DCVs}^{(5,6)}$

Assim, o presente estudo se propõe a investigar não diretamente os fatores de risco às DCVs, mas sim, as variáveis que possam estar atreladas a estes tipos de manifestações nefastas à saúde. Portanto, os objetivos traçados são: descrever e explorar o perfil da aptidão física relacionada à saúde (ApFRS); o nível de atividade física habitual (AFh); o número de horas de assistência de televisão (Hrs.TV); e a prevalência de tabagismo e etilismo em crianças e jovens escolares da cidade de Barra do Ribeiro/RS-Brasil, divididos por idades (10 a 14 anos) e sexo (rapazes e moças).

\section{METODOLOGIA}

$\mathrm{O}$ projeto que originou a pesquisa foi proposto para a Secretaria Municipal de Educação, Cultura e Esportes (SMECE) do município de Barra do Ribeiro/RS. Assim, o projeto foi realizado em quatro escolas: três escolas da rede municipal de ensino e uma escola da rede privada.

O município de Barra do Ribeiro pertence à microrregião de Camaquã, que é localizada na região metropolitana de Porto Alegre, Rio Grande do Sul, Brasil. Sua área total é de $730,82 \mathrm{Km}^{2}$. De acordo com dados do Instituto Brasileiro de Geografia e Estatística (IBGE) (7), o município possui aproximadamente 11.845 habitantes. Da totalidade de habitantes, estima-se que $25 \%$ a $30 \%$ residem na zona rural da cidade. Segundo informações do site da Fundação de Economia e Estatística (FEE)(8), a população é considerada na sua maioria, pobre, apresentando índices baixos de desenvolvimento humano. Este perfil econômico pode ser responsável por um estilo de vida bastante limitado de atividades socioculturais, desportivas e de lazer. Inclusive, no município não existem ofertas destes tipos de atividades como cinemas, teatro, shows, escolinhas de formação esportiva ou programas de promoção à saúde para a população de crianças e jovens.

\section{AMOSTRA}

O estudo procurou atender ao maior número de crianças e jovens escolares, dentro da faixa etária de 10 a 14 anos, que estavam matriculados nas quatro escolas participantes do projeto. Assim, a amostra pode ser caracterizada como intencional.

Diante destes critérios, ressalva-se que o número de rapazes avaliados no presente estudo foi de 124 e o número de moças avaliadas foi de 116 . Pode ser observada na TABELA 1, a distribuição dos escolares avaliados no presente estudo conforme idade (10 a 14 anos) e sexo (rapazes e moças).

TABELA 1. DISTRIBUIÇÃO DOS ESCOLARES CONFORME IDADE E SEXO.

\begin{tabular}{lllllll}
\hline & 10 & 11 & 12 & 13 & 14 & Total \\
\hline Rapazes & 22 & 39 & 23 & 21 & 19 & 124 \\
Moças & 28 & 29 & 32 & 14 & 13 & 116 \\
\hline Total & 50 & 68 & 56 & 35 & 32 & 240 \\
\hline
\end{tabular}

\section{PROCEDIMENTOS DE COLETAS DE DADOS}

A abordagem inicial da avaliação foi feita por meio da aplicação de um questionário, o qual foi respondido pelos alunos dentro da própria sala de aula. Num segundo momento foram realizadas as avaliações físicas com os alunos. Todas estas avaliações ocorreram ao longo dos meses de novembro e dezembro de 2008, internamente nas escolas, com o apoio dos respectivos professores de educação física.

O questionário foi composto por três unidades. $\mathrm{Na}$ unidade I os alunos responderam informações referentes aos dados pessoais, para o respectivo controle interno da pesquisa. $\mathrm{Na}$ unidade II, os alunos responderam informações referentes aos hábitos de tabagismo e etilismo. $O$ tabagismo foi identificado por meio de duas questões fechadas retiradas do Global School-Based Student Health Survey (GSHS, 2004) (9) $^{(9)}$ trabalho que têm sido amplamente utilizados em levantamentos epidemiológicos. $O$ etilismo foi avaliado por meio de duas 
questões adaptadas do questionário Cage, que é um questionário para a detecção precoce do etilismo, elaborado por Ewing e Rouse (1970) apud Masur $(1985)^{(10)}$. Na unidade III os alunos responderam informações referentes aos hábitos de atividade física. Para esta unidade foi utilizado o Physical Activity Questionnaire for Older Children (PAQ-C).

Este questionário investigativo da prática de atividade física habitual de crianças entre oito e 14 anos foi proposto inicialmente por Crocker, et al. (1997)(11) e Kowalski et al. (1997)(12). O questionário foi traduzido e modificado para a língua portuguesa brasileira com o objetivo de excluir algumas atividades físicas e esportivas não praticadas no Brasil ${ }^{(13,14)}$. O questionário investiga a prática de atividade física habitual de crianças e jovens nos sete dias anteriores ao seu preenchimento. Ele é composto por nove questões sobre a prática de esportes e jogos, de atividades físicas na escola e no lazer, incluindo o final de semana. Cada questão tem o valor de um a cinco e o escore final é obtido pela média das questões, representando o intervalo de muito sedentário (um) a muito ativo (cinco). Os escores dois, três e quatro indicam as categorias: sedentário, moderadamente ativo e ativo, respectivamente. Neste questionário há ainda uma décima pergunta na qual as crianças e jovens respondem qual o número de horas de assistência de televisão em média, por dia.

Nas avaliações físicas foram realizadas as medidas de massa corporal e estatura; as dobras cutâneas do tríceps e da perna; a circunferência da cintura; e os testes de flexibilidade (sentar e alcançar sem banco de Wells), força/ resistência abdominal (sit up's) e resistência geral (caminhada/corrida de nove minutos). Com as medidas de estatura e massa corporal foi calculado o Índice de Massa Corporal (IMC) dos escolares.

Para avaliar as medidas de massa corporal $(\mathrm{Kg})$ e estatura $(\mathrm{cm})$, e os testes de flexibilidade $(\mathrm{cm})$, força/resistência abdominal (repetições máximas por minuto) e resistência geral (metros), foram utilizadas as medidas e testes do
Projeto Esporte Brasil( ${ }^{(15)}$. A instrumentalização necessária para estas medidas e testes foi: balança portátil com precisão de $500 \mathrm{~g}$ (massa corporal), fita métrica inextensível com precisão em $0,5 \mathrm{~cm}$ (estatura e flexibilidade), colchonetes (força/resistência abdominal), um espaço de corrida - quadra esportiva - (caminhada/corrida de nove minutos), fita crepe para demarcações e ficha de avaliação para anotações não somente destas medidas e testes, mas de toda a avaliação física.

Para as dobras cutâneas do tríceps $(\mathrm{mm})$ e da perna $(\mathrm{mm})$ seguiram-se os protocolos de medidas da International Society for the Advancement of Khinantropometry ${ }^{(16)}$. O instrumento para a coleta de dados foi o plicômetro científico da marca CESCORF, com precisão de 0,1 $\mathrm{mm}$.

$\mathrm{Na}$ circunferência da cintura $(\mathrm{cm})$ utilizouse o protocolo de Kartzmarzik et al. $(2004)^{(17)}$ e o instrumento necessário foi a fita métrica inextensivel com precisão de $0,5 \mathrm{~cm}$.

Em todas estas avaliações físicas os alunos foram orientados para trajarem camiseta, bermuda, meias e tênis adequado para a prática esportiva.

\section{TRATAMENTO ESTATÍSTICO DOS DADOS}

$\mathrm{Na}$ análise dos dados, primeiramente foi realizado um estudo exploratório no intuito de avaliar os pressupostos essenciais da análise paramétrica, e para a identificação dos indivíduos considerados outliers severos. Como houve a confirmação da normalidade das distribuições e a não ocorrência de indivíduos considerados outliers, partiu-se para o tratamento descritivo das variáveis de análise (ApFRS, AFh), com os individuos divididos por idades (10 a 14 anos) e sexo (rapazes e moças). Nesta análise descritiva foram utilizados os valores de médias e desvios-padrões. Utilizaram-se ainda os valores de freqüência de ocorrência para as variáveis: tabagismo e etilismo. Os dados foram tabulados e tratados no software Statistical Package for the Social Sciences (SPSS) for Windows, versão 17.0 . 


\section{CONSENTIMENTO ÉTICO}

A pesquisa foi conduzida dentro dos padrões éticos exigidos pela Declaração de Helsinque de 1964 e de acordo com a resolução 196/96 do Ministério da Saúde. Todos os alunos participantes da pesquisa receberam um Termo de Consentimento Livre e Esclarecido através das diretoras das escolas. Estes Termos foram levados para casa e assinados, ou não, pelos seus respectivos pais e/ou responsáveis. Os alunos participantes também tiveram que assinar o Termo. As escolas participantes do estudo bem como a Secretaria Municipal de Educação, Cultura e Esportes (SMECE) do município de Barra do Ribeiro/RS receberam um Termo de Consentimento Livre e Esclarecido Institucional, onde as respectivas diretoras e secretária tiveram que assinar. 0 projeto que gerou o devido estudo foi aprovado pelo Comitê de Ética em Pesquisas (CEP) da Universidade do Vale do Paraiba (UNIVAP) sob Protocolo No. H72/CEP/2008.

\section{RESULTADOS}

$\mathrm{Na}$ TABELA 2 foram descritos os valores de média e desvio-padrão das variáveis relacionadas à aptidão física e saúde (ApFRS), e atividade física habitual (AFh) dos rapazes divididos por idades (10 a 14 anos): massa corporal, estatura, IMC, dobras cutâneas (DC) de tríceps e biceps, circunferência $(C)$ de cintura, flexibilidade, força/resistência (F/R) abdominal, resistência geral, Physical Activity Quetionnaire for Older Children (PAQ-C) e horas de assistência de televisão (Hrs. TV).

Em relação à massa corporal, estatura, IMC, circunferência de cintura, força/resistência abdominal e resistência geral, foi possivel observar um incremento nos valores médios dos 10 para os 14 anos de idade. Na flexibilidade, ocorreu o contrário, ou seja, dos 10 para os 14 anos de idade houve um decréscimo nos valores de média. Nas variáveis de dobras cutâneas do tríceps e perna, não houve um padrão de incremento ou decréscimo de médias. O que ocorreu, foi uma variação nos valores observados entre $11 \mathrm{~mm}$ e $14 \mathrm{~mm}$ de espessura para a variável DC tríceps e entre $10 \mathrm{~mm}$ e $13 \mathrm{~mm}$ para a variável DC perna.

Nos escores do PAQ-C e nas horas de assistência de TV foi possivel observar também uma variação nos escores do questionário e nas horas de assistência de TV entre as idades 10 e 14 anos. Especificamente nas idades 10,13 e 14 anos, os valores médios dos escores do PAQ-C classificam os individuos em moderadamente ativos e nas idades 11 e 12 anos, os valores médios classificam os indivíduos em sedentários. Por sua vez, nas horas de assistência de TV a variação ficou próxima ao valor de três horas e meia para todas as idades.

TABELA 2. DESCRIÇÃO DAS MÉDIAS E (DESVIOSPADRÕES) DO PERFIL DAAPFRS E AFH DOS RAPAZES DIVIDIDOS POR IDADES.

\begin{tabular}{|c|c|c|c|c|c|}
\hline & 10 anos & 11 anos & 12 anos & 13 anos & 14 anos \\
\hline \multirow[t]{2}{*}{ Massa Corporal } & 36,88 & 42,37 & 48,09 & 49,83 & 55,98 \\
\hline & $( \pm 7,41)$ & $( \pm 10,76)$ & $( \pm 14,59)$ & $( \pm 8,69)$ & $( \pm 13,22)$ \\
\hline \multirow[t]{2}{*}{ Estatura } & 147,68 & 151,28 & 157,26 & 161,09 & 169,00 \\
\hline & $( \pm 5,84)$ & $( \pm 7,43)$ & $( \pm 9,96)$ & $( \pm 9,78)$ & $( \pm 9,81)$ \\
\hline \multirow[t]{2}{*}{ IMC } & 16,80 & 18,32 & 19,08 & 19,27 & 19.46 \\
\hline & $( \pm 2,64)$ & $( \pm 3,79)$ & $( \pm 3,89)$ & $( \pm 2,92)$ & $( \pm 3,60)$ \\
\hline \multirow[t]{2}{*}{ DC Triceps } & 12,52 & 14,66 & 14,61 & 12,78 & 11,93 \\
\hline & $( \pm 5,12)$ & $( \pm 6,17)$ & $( \pm 7,17)$ & $( \pm 5,44)$ & $( \pm 4,24)$ \\
\hline \multirow[t]{2}{*}{ DC Perna } & 10,92 & 12,38 & 13,49 & 13,23 & 11.90 \\
\hline & $( \pm 4,43)$ & $( \pm 5,53)$ & $( \pm 6,98)$ & $( \pm 7,07)$ & $( \pm 4,80)$ \\
\hline \multirow[t]{2}{*}{ C. Cintura } & 64,40 & 68,19 & 70,04 & 70,83 & 71,86 \\
\hline & $( \pm 8,13)$ & $( \pm 10,90)$ & $( \pm 12,05)$ & $( \pm 8,90)$ & $( \pm 8,45)$ \\
\hline \multirow{2}{*}{ Flexibilidade } & 34,72 & 32,89 & 32,43 & 30,57 & 30,10 \\
\hline & $( \pm 7,84)$ & $( \pm 6,93)$ & $( \pm 9,98)$ & $( \pm 9,27)$ & $( \pm 10,95)$ \\
\hline \multirow[t]{2}{*}{ F/R Abdominal } & 30,36 & 32,79 & 32,93 & 33,33 & 35,10 \\
\hline & $( \pm 10,67)$ & $( \pm 8,10)$ & $( \pm 7,61)$ & $( \pm 9,46)$ & $( \pm 9,47)$ \\
\hline \multirow[t]{2}{*}{ Res. Geral } & 1250,68 & 1316,66 & 1343,04 & 1410,47 & 1442,63 \\
\hline & $( \pm 170,11)$ & $( \pm 171,39)$ & $( \pm 196,27)$ & $( \pm 159,43)$ & $( \pm 143,21)$ \\
\hline \multirow[t]{2}{*}{$P A Q-C$} & 3,13 & 2,80 & 2,99 & 3,15 & 3,21 \\
\hline & $( \pm 0,83)$ & $( \pm 0,80)$ & $( \pm 0,83)$ & $( \pm 0,90)$ & $( \pm 0.71)$ \\
\hline \multirow[t]{2}{*}{ Hrs. TV } & 3,22 & 3,64 & 3,56 & 3,33 & 3,57 \\
\hline & $( \pm 0,92)$ & $( \pm 1,56)$ & $( \pm 1,19)$ & $( \pm 1,27)$ & $( \pm 0,83$ \\
\hline
\end{tabular}

$\mathrm{Na}$ TABELA 3 foram descritos os valores de média e desvio-padrão das variáveis relacionadas à aptidão física e saúde (ApFRS), e atividade física habitual (AFh) das moças divididas por idades (10 a 14 anos). 
TABELA 3. DESCRIÇÃO DAS MÉDIAS E (DESVIOSPADRÕES) DO PERFIL DAApFRS E AFh DAS MOÇAS DIVIDIDAS POR IDADES.

\begin{tabular}{lccccc}
\hline & 10 anos & 11 anos & 12 anos & 13 anos & 14 anos \\
\hline Massa Corporal & 38,23 & 41,69 & 43,76 & 47,35 & 53,28 \\
& $( \pm 7,76)$ & $( \pm 11,07)$ & $( \pm 9,82)$ & $( \pm 9,11)$ & $( \pm 9,58)$ \\
\cline { 2 - 6 } Estatura & 146,14 & 150,31 & 152,17 & 156,85 & 161,38 \\
& $( \pm 6,65)$ & $( \pm 8,68)$ & $( \pm 28,35)$ & $( \pm 6,23)$ & $( \pm 5,22)$ \\
\cline { 2 - 6 } IMC & 17,76 & 18,13 & 18,39 & 19,20 & 20,43 \\
& $( \pm 2,55)$ & $( \pm 3,26)$ & $( \pm 3,45)$ & $( \pm 3,35)$ & $( \pm 3,25)$ \\
\cline { 2 - 6 } DC Triceps & 14,11 & 14,18 & 13,97 & 14,16 & 15,72 \\
& $( \pm 4,70)$ & $( \pm 4,22)$ & $( \pm 5,07)$ & $( \pm 4,16)$ & $( \pm 4,52)$ \\
\cline { 2 - 6 } DC Perna & 14,13 & 13,88 & 13,11 & 14,80 & 15,16 \\
& $( \pm 4,22)$ & $( \pm 4,27)$ & $( \pm 5,52)$ & $( \pm 3,94)$ & $( \pm 4,17)$ \\
\cline { 2 - 6 } C. Cintura & 64,17 & 65,09 & 65,96 & 67,42 & 68,75 \\
& $( \pm 6,73$ & $( \pm 10,46)$ & $( \pm 7,86)$ & $( \pm 9,00)$ & $( \pm 9,33)$ \\
\cline { 2 - 6 } Flexibilidade & 39,57 & 38,65 & 37,15 & 35,14 & 34,84 \\
& $( \pm 8,08)$ & $( \pm 8,64)$ & $( \pm 7,66)$ & $( \pm 5,92)$ & $( \pm 10,08)$ \\
\cline { 2 - 6 } F/R Abdominal & 27,39 & 27,86 & 26,78 & 27,21 & 26,00 \\
& $( \pm 10,00)$ & $( \pm 12,07)$ & $( \pm 8,06)$ & $( \pm 5,69)$ & $( \pm 11,15)$ \\
\cline { 2 - 6 } Res. Geral & 1229,64 & 1238,62 & 1279,06 & 1335,09 & 1386,92 \\
& $( \pm 123,27)$ & $( \pm 157,08)$ & $( \pm 186,77)$ & $( \pm 184,37)$ & $( \pm 191,07)$ \\
\cline { 2 - 6 } PAQ-C & 2,87 & 2,62 & 2,76 & 2,57 & 2,65 \\
& $( \pm 0,57)$ & $( \pm 0,48)$ & $( \pm 0,60)$ & $( \pm 0,84)$ & $( \pm 0,83$ \\
\cline { 2 - 6 } Hrs. TV & 3,13 & 4,17 & 3,87 & 3,97 & 3,38 \\
& $( \pm 1,08)$ & $( \pm 1,22)$ & $( \pm 1,18)$ & $( \pm 1,64)$ & $( \pm 1,60)$ \\
\hline
\end{tabular}

Ao observar esta tabela, se percebe que é possível realizar análises similares às feitas para os rapazes. Em relação à massa corporal, estatura, IMC, circunferência de cintura, força/resistência abdominal e resistência geral, se percebe um incremento nos valores médios dos 10 para os 14 anos de idade; e na flexibilidade ocorreu também o contrário.

Nas variáveis de dobras cutâneas do triceps e perna, assim como nos rapazes, não houve um padrão de incremento ou decréscimo de médias. O que ocorreu, foi uma variação nos valores observados entre $13 \mathrm{~mm}$ e $15 \mathrm{~mm}$ de espessura para as variáveis DC triceps e DC perna. No PAQ-C e nas horas de assistência de TV também foi possivel observar uma variação nos escores do questionário e nas Hrs. TV entre as idades 10 e 14 anos. Entretanto, diferentemente dos rapazes, as moças, em todas as idades (10 a 14 anos) tiveram os valores médios dos escores do PAQ-C classificados na categoria sedentários. $E$, nas horas de assistência de TV a variação ficou próxima ao valor de três horas e meia para as idades $10,12,13$ e 14 anos, com exceção da idade 12 anos cuja média foi de 4,14 horas.
Nas FIGURAS 1 e 2 estão retratados os valores de prevalência de tabagismo e etilismo, respectivamente; agrupando todas as idades (10 a 14 anos) e dividindo os escolares apenas por sexo (rapazes e moças). Em relação ao tabagismo (FIGURA 1) foi possivel observar 10 rapazes (8,06\% - 1 rapaz de 10 anos, 3 rapazes de 11 anos, 1 rapaz de 12 anos, 2 rapazes de 13 anos e 3 de 14 anos) adeptos, e 8 moças $(6,90 \%$ - 3 moças de 12 anos, 2 de 13 anos e 3 de 14 anos) adeptas ao hábito de fumar. $E$ foi possível observar 41 rapazes $(33,06 \%$ - 6 rapazes de 10 anos, 9 de 11 anos, 10 de 12 anos, 8 de 13 anos e 8 de 14 anos) e 32 moças (27,59\% - 4 moças de 10 anos, 6 de 11 anos, 5 de 12 anos, 7 de 13 anos e 10 de 14 anos) cujo questionário aplicado discriminouos como adeptos ao hábito de ingerir bebidas alcoólicas (FIGURA 2).

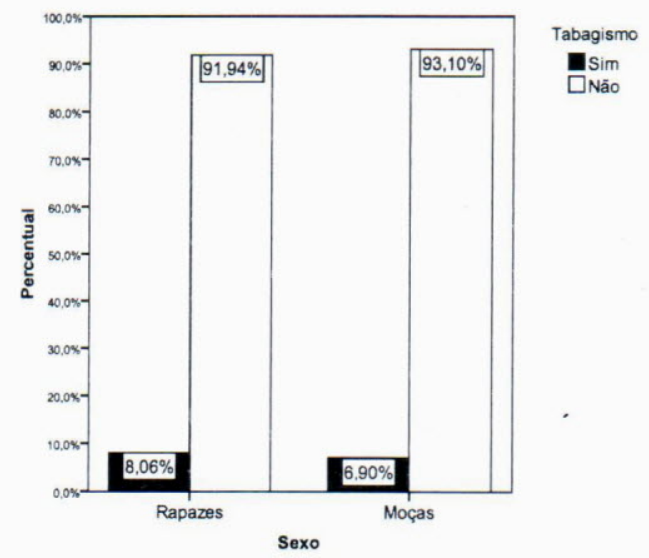

FIGURA 1. PREVALÊNCIA DE TABAGISMO NOS ESCOLARES DE AMBOS OS SEXOS (10 A 14 ANOS).

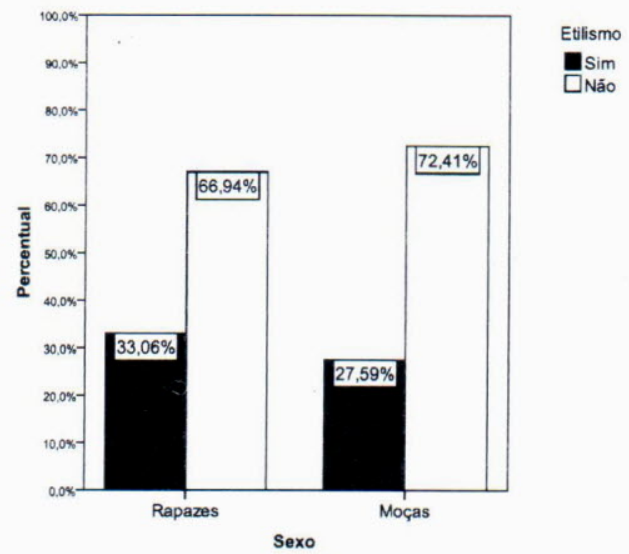

FIGURA 2. PREVALÊNCIA DE ETILISMO NOS ESCOLARES DE AMBOS OS SEXOS (10 A 14 ANOS). 


\section{DISCUSSÃO}

Partindo do princípio de que o estudo propôs a descrição do perfil da aptidão física relacionada à saúde e dos hábitos de vida em crianças e jovens escolares da cidade de Barra do Ribeiro/RS-Brasil, divididos por idades (10 a 14 anos) e sexo (rapazes e moças), torna-se possível considerar que o objetivo principal foi alcançado. Diferentemente da grande maioria dos estudos científicos publicados nas Ciências do Movimento Humano e da Saúde, este estudo não propôs como objetivo comparar os perfis entre as diferentes idades (10 a 14 anos) e sexo (rapazes e moças). Detemo-nos apenas à descrição de dados. Fato este embasado no pressuposto da não aleatoriedade da amostra de estudo, ou seja, a amostra do presente estudo não é probabilística, que é o pressuposto primordial para a realização das inferências estatísticas. Neste estudo, procurou-se adequar ao coerente, ao pressuposto exato, descrito em diferentes literaturas perfilhadas ${ }^{(1-4)}$ Entretanto, não é de se minimizar o cientificismo do presente estudo. Ele apenas contempla, configura outra realidade de análise, ou seja, a análise descritiva de fenômenos em uma determinada população de crianças e jovens.

Neste sentido, a discussão dos resultados encontrados será realizada a partir das evidências de algumas pesquisas, nacionais e internacionais, que tiveram objetivos similares ao do presente estudo. É importante ressalvar que principalmente as possiveis diferenças metodológicas entre o presente estudo e os estudos que servirão como base para a discussão podem ser consideradas como uma possível limitação. Sem ponderar que os diferentes estudos avaliaram distintas populações de crianças e jovens, em diferentes contextos sociais e culturais e em diferentes momentos (anos), o que também deve ser considerado como limitação das análises.

Fundamentalmente, ao observar os resultados retratados nas TABELAS 2 e 3, torna-se possivel analisar que tanto os rapazes quanto as moças apresentaram nas variáveis de massa corporal, estatura, IMC, circunferência de cintura, força/resistência abdominal e resistência geral um padrão de desenvolvimento ao longo das idades (10 a 14 anos). Este comportamento de crescimento e desenvolvimento somatomotor pode ser elucidado pelo fato das crian- ças e jovens estarem em uma faixa etária onde ocorrem muitas transformações corporais devido principalmente à ação hormonal, que tem sua secreção aumentada durante este período.

Os resultados do presente estudo, principalmente os que tangem as variáveis motoras (força/resistência abdominal e resistência geral), são corroborados pelos estudos clássicos desenvolvidos por Farinatti $(1995)^{(18)}$, Malina e Bouchard (2002) ${ }^{(19)}$ e Gallahue e Ozmun $(2005)^{(20)}$. Estes autores enfatizam que durante esta faixa etária, juntamente com o rápido crescimento somático, há o aumento dos órgãos vitais como o coração e pulmões, há o aumento da massa muscular, da capacidade de captação e uso do oxigênio, os quais têm influência direta na melhora da capacidade cardiorrespiratória e de força muscular. Especificamente o desenvolvimento da resistência geral, mensurada pelo teste de caminhada/corrida de nove minutos, pode também estar associada à meIhora do padrão motor da marcha, ou seja, à economia de corrida, que tende a ocorrer em grande escala durante os periodos da infância e adolescência.

Em relação à variável de flexibilidade foi observado no presente estudo que os rapazes e as moças apresentaram um decréscimo nos valores de média dos 10 para os 14 anos de idade. Fato este não comum nos achados de outros estudos. De acordo com Malina e Bouchard (2002) $)^{(19)}$, dos sete aos 10 anos nas moças e dos sete aos 11-12 anos nos rapazes, há uma redução nos níveis de flexibilidade. A partir dos 10 anos para as meninas e a partir dos 11-12 anos para os meninos, a capacidade de flexibilidade tende a aumentar até por volta dos 14-15 anos. Em outro estudo foi encontrado um comportamento ondulatório nos níveis de flexibilidade dos 10 aos 14 anos, sendo que os ganhos máximos na capacidade foram por volta dos 12-13 anos de idade para ambos os sexos ${ }^{(21)}$.

Nas variáveis de dobras cutâneas do tríceps e perna não houve um padrão de incremento ou decréscimo de médias, tanto para os rapazes quanto para as moças. O que ocorreu, foi uma variação nos valores observados entre $11 \mathrm{~mm}$ e $14 \mathrm{~mm}$ de espessura (rapazes) e $13 \mathrm{~mm}$ e $15 \mathrm{~mm}$ (moças) para a variável DC triceps; e entre $10 \mathrm{~mm}$ e $13 \mathrm{~mm}$ (rapazes) e $13 \mathrm{~mm}$ e $15 \mathrm{~mm}$ (moças) para a variável DC perna. De uma forma geral, as pesquisas demonstram que 
não há um padrão de incremento ou decréscimo em relação ao acúmulo de gordura corporal subcutânea, principalmente nesta faixa etária de estudo (10 a 14 anos). Esta variável está intimamente associada ao comportamento sedentário ou não do indivíduo, aos seus hábitos alimentares e ao componente genético. Apesar disto, se observa que as meninas tendem a ter um acúmulo maior de gordura subcutânea em relação aos meninos, o que aparentemente ocorreu no presente estudo; e este acúmulo tende a se localizar nas regiões central e do tronco no corpo humano ${ }^{(22)}$. Entretanto, para verificar este acúmulo nestas regiões seria necessário mensurar outras dobras cutâneas não mensuradas neste estudo como, por exemplo, dobra cutânea abdominal, supra-ilíaca e subescapular.

Para as variáveis, nível de atividade física habitual e horas de assistência de TV, tanto para os rapazes quanto para as moças não houve um padrão nos resultados. Foi encontrado no presente estudo que aos 10,13 e 14 anos de idade os rapazes tiveram os valores médios dos escores do PAQ-C classificados com moderadamente ativos e nas idades 11 e 12 anos, os valores médios classificados em sedentários. As moças, em todas as idades (10 a 14 anos) tiveram os valores médios dos escores do PAQ-C classificados na categoria sedentários. Por sua vez, nas horas de assistência de TV a variação ficou próxima ao valor de três horas e meia para todas as idades e ambos os sexos. Com exceção das moças de 12 anos de idade cuja média foi de 4,14 horas de assistência de TV.

Em relação aos escores do PAQ-C, as médias encontradas para as idades e sexos no presente estudo são similares aos achados nos estudos que validaram o respectivo instrumento na população internacional| ${ }^{(11,12)}$. E são diferentes dos achados nos estudos que validaram o instrumento na população nacional ${ }^{(13,14)}$. Nas horas de assistência de TV a literatura nacional apresenta uma variação entre 3,6 e 4,4 horas por dia para os meninos e 3,6 e 4,9 horas por dia para as meninas $(13,14,23,24)$. E na literatura internacional os autores relatam que a média varia entre 2 e 3 horas diárias nos $E \mathrm{~A}^{(25)}$ e 2,1 e 2,3 horas por dia no Canadá(26), para rapazes e moças em idade escolar.

Por fim, são destacados os resultados encontra- dos através da aplicação dos questionários de tabagismo e etilismo. Por meio destes, foi possivel observar que $8,06 \%$ dos rapazes e $6,90 \%$ das moças têm o hábito de fumar; e $33,06 \%$ dos rapazes e $27,59 \%$ das moças têm o hábito de ingerir bebidas alcoólicas. Isto representa uma totalidade de 10 rapazes e 8 moças fumantes; e 41 rapazes e 32 moças que ingerem bebidas alcoólicas regularmente. Ou seja, 18 escolares (tabagismo) e 73 escolares (etilismo) do total de 240, já possuem ao menos um fator de risco às doenças cardiovasculares e cancerigenas. Fato alarmante tendo em vista que eles ainda se encontram em uma faixa etária inicial da vida.

\section{CONCLUSÃO}

O presente estudo descritivo exploratório permitiu-nos conhecer em que estado se encontra uma razoável parcela de crianças e jovens (10 a 14 anos rapazes e moças) do município de Barra do Ribeiro/ $\mathrm{RS}$, em relação à aptidão física e saúde e aos hábitos de vida. Acredita-se na possibilidade destes achados servirem de base teórica para outros pesquisadores da área abordada bem como para auxiliar no incentivo à aprovação de um projeto social na cidade. Destaca-se também que é de fundamental importância a continuidade dos estudos de perfil para o amplo conhecimento da realidade das crianças e jovens nas mais diferentes regiões do Brasil.

\section{REFERÊNCIAS BIBLIOGRÁFICAS}

1- Callegari-Jacques SM. Bioestatística: princípios e aplicações. Porto Alegre: Artmed, 2003.

2- Katz DL, Elmore JG, Jekel JF. Epidemiologia, Bioestatística e Medicina Preventiva. $2^{a}$. ed. Porto Alegre: Artmed, 2005.

3- Thomas JR, Nelson JK, Silverman SJ. Métodos de Pesquisa em Educação Física. 5a ${ }^{a}$. ed. Porto Alegre: Artmed, 2007

4- Gaya ACA, Garlipp DC, Silva M. Ciências do Movimento Humano: Introdução à Metodologia da Pesquisa. Porto Alegre: Artmed, 2008.

5- Ribeiro JC, Guerra S, Oliveira J, et al. Physical activity and biological risk factor clustering in pediatric population. Prev Med 2004; 39; 596-601. 
6- Reed KE, Warburton DER, McKay HA. Determining cardiovascular disease risk in elementary school children: developing a healthy heart score. J Sports Med Sci 2007; 6: 142-148.

7- IBGE - Instituto Brasileiro de Geografia e Estatística. http://www.ibge.org.br Acessado em 16 de maio de 2010.

8- FEE - Fundação de Economia e Estatística. http:// www.fee.tche.br Acessado em 16 de maio de 2010.

9- GSHS. Global School-Based Student Health Survey. 2004. Disponível em http://www.cdc.gov/gshs Acessado em 18 de outubro de 2008.

10- Masur J. Deteç̧ão precoce do alcoolismo em clínica médica através do CAGE. J Bras Psiq 1985; 34: 31-34.

11- Crocker PR, Bailey DA, Faulkner RA, Kowalski KC, McGrath R. Measuring general levels of physical activity: preliminary evidence for the Physical Activity Questionnaire for Older Children. Med Sci Sports Exerc 1997; 29: 1344-1349.

12- Kowalski KC, Crocker PR, Faulkner RA. Validation of the physical activity questionnaire for older children. Ped Exerc Sci 1997; 9: 174-186.

13- Rosendo da Silva RC. Coronary heart disease risk factors and health-related fitness of adolescents in Niterói, Rio de Janeiro, Brazil. [Tese de Doutorado]. Michigan State University, East Lansing, Michigan, EUA, 1998.

14- Rosendo da Silva RC, Malina RM. Nível de atividade física em adolescentes do município de Niterói, Rio de Janeiro, Brasil. Cad Saúde Pública 2000; 16(4): 10911097

15- PROESP-BR - Projeto Esporte Brasil: Observatório permanente de indicadores de crescimento e desenvolvimento corporal, motor e estado nutricional de crianças e jovens de 7 a 17 anos. Manual de Aplicação de Medidas e Testes, Normas e Critérios de Avaliação. Gaya ACA (ed.), 2007. Disponível em http://www. proesp.ufrgs. br Acessado em 21 de junho de 2008.

16- ISAK. International Standards for Anthropometric Assessment. Marfell-Jones M, Olds T, Stewart A, Carter JEL (eds.). ISAK: Potchefstroom, 2006.

17- Kartzmarzyk PT, Srinivasan SR, Chen W, et al. Body mass index, waist circumference, and clustering of cardiovascular disease risk factor in a biracial sample of children and adolescents. Pediatrics. 2004; 114:198-205.
18- Farinatti PTV. Criança e atividade física. Rio de Janeiro: Sprint, 1995.

19- Malina RM, Bouchard C. Atividade física do atleta jovem: do crescimento à maturação. São Paulo: Roca, 2002.

20- Gallahue DL, Ozmun JC. Compreendendo o Desenvolvimento Motor: bebês, crianças, adolescentes e adultos. $3^{a}$. ed. São Paulo: Phorte, 2005.

21- Bergmann GG. Crescimento somático, aptidão física relacionada à saúde e estilo de vida de escolares de 10 a 14 anos: um estudo longitudinal. [Dissertação de Mestrado]. Escola de Educação Física, Universidade Federal do Rio Grande do Sul, Porto Alegre, Brasil, 2006.

22- Lohman TG. The use of skinfold to estimate body fatness on children and youth. JOPERD 1987; 58(9): 98102.

23- Andrade D, Araújo T, Figueira A, Matsudo VK. Comparison of physical activity involvement in Brazilian teenagers. In.: Physical activity, sport and health. The International Pre-Olympic Scientific Congress (ed). Dallas: Institute for Aerobics Research, 1996.

24- Matsudo SM, Matsudo VK, Andrade D, Rocha JR. Physical and time spent watching TV in children from low socioeconomic region. Med Sci Sports Exerc 1997; 29(supl): 237.

25- Pate R, Long B, Heath G. Descriptive epidemiology of physical activity in adolescents. Ped Exerc Sci 1994; 6: 434-447.

26- Kartzmarzyk PT, Malina R, Song T, Bouchard C. Television viewing, physical activity and health-related fitness of youth in the Québec Family Study. J Adolesc Health 1998; 23: 318-325.

Endereço para correspondência:

Rafael Abeche Generosi

Programa de Pós-Graduação em Ciências do movimento Humano (PPGCMH), Escola de Educação Física (ESEF), Universidade Federal do Rio Grande do Sul (UFRGS).

Rua Felizardo, 750 - Sala 201 - Bairro Jardim Botânico - Porto Alegre, RS, Brasil

CEP: $90690-200$

Fone: (51) 3308-5819 / (54) 9176-5519

E-mails: rafaelgenerosi@gmail.com 